\title{
Impact of dispersion on pulse dynamics in chirped-pulse fiber lasers
}

\author{
M. Baumgartl · B. Ortaç · J. Limpert • A. Tünnermann
}

Received: 5 March 2012 / Published online: 26 April 2012

C) Springer-Verlag 2012

\begin{abstract}
We report on a systematic study of an environmentally stable mode-locked Yb-doped fiber laser operating in the chirped-pulse regime. The linear cavity chirped-pulse fiber laser is constructed with a saturable absorber mirror as nonlinear mode-locking mechanism and a nonlinearity-free transmission-grating-based stretcher/compressor for dispersion management. Mode-locked operation and pulse dynamics from strong normal to strong anomalous total cavity dispersion in the range of +2.5 to $-1.6 \mathrm{ps}^{2}$ is experimentally studied. Strongly positively chirped pulses from $4.3 \mathrm{ps}$ $\left(0.01 \mathrm{ps}^{2}\right)$ to $39 \mathrm{ps}\left(2.5 \mathrm{ps}^{2}\right)$ are obtained at normal netcavity dispersion. In the anomalous dispersion regime, the laser generates average soliton feature negatively chirped pulses with autocorrelation pulse durations from $0.8 \mathrm{ps}$ $\left(-0.07 \mathrm{ps}^{2}\right)$ to $3.9 \mathrm{ps}\left(-1.6 \mathrm{ps}^{2}\right)$. The lowered peak power due to the pulse stretching allows one to increase the double pulse threshold. Based on the numerical simulation, different regimes of mode locking are obtained by varying the intra-cavity dispersion, and the characteristics of average
\end{abstract}

M. Baumgartl ( $₫)$ · J. Limpert · A. Tünnermann

Institute of Applied Physics, Friedrich-Schiller-Universität Jena, Albert-Einstein-Str. 15, 07745 Jena, Germany

e-mail: martin.baumgartl@uni-jena.de

Fax: +49-3641-947802

M. Baumgartl · J. Limpert · A. Tünnermann

Helmholtz-Institute Jena, Max-Wien-Platz 1, 07743 Jena,

Germany

B. Ortaç

UNAM-Institute of Materials Science and Nanotechnology, Bilkent University, 06800 Bilkent, Ankara, Turkey

A. Tünnermann

Fraunhofer Institute for Applied Optics and Precision Engineering, Albert-Einstein-Str. 7, 07745 Jena, Germany soliton, stretched-pulse, wave-breaking-free and chirpedpulse regimes are discussed.

\section{Introduction}

Mode-locked, rare-earth-doped fiber lasers are an ideal source for numerous ultrafast applications, as they possess a large amplification bandwidth and allow for robust and cost-effective implementation. In particular, the use of polarization maintaining fibers lead to environmentally stable, compact and highly efficient laser systems [1,2].

Furthermore, due to the tight confinement the intense light field of an ultrashort pulse experiences a strong nonlinear response during fiber propagation, which, together with the high single-pass gain, leads to a rich nonlinear dynamic inside the fiber laser resonator, which strongly differs from other mode-locked solid state lasers. Hence, besides having been driven by application demands, passively mode-locked fiber lasers themselves have been an interesting research area and a variety of different operation regimes (Fig. 1) have been reported.

Pulse formation, evolution and shaping is, besides by the mode-locking mechanism, primarily governed by the intracavity dispersion and its interplay with Kerr nonlinearity. In lasers entirely constructed of negative group velocity dispersion (GVD) fibers, conventional chirp-free solitons form and propagate steadily along the cavity [3]. Soliton characteristics are also observed in lasers with a non-uniform dispersion map operating in the so-called dispersion-managed soliton regime [4]. Close to zero net-cavity dispersion alternating, highly dispersive fiber sections cause significant variations of both pulse duration and chirp during each resonator round trip. This way pulse characteristics can be tailored and higher energies and shorter pulse durations be- 
Fig. 1 Overview over the different pulse regimes in passively mode-locked $\mathrm{Yb}$-fiber lasers and their appellation in literature, the light gray arrow marks the range covered by our experimental setup

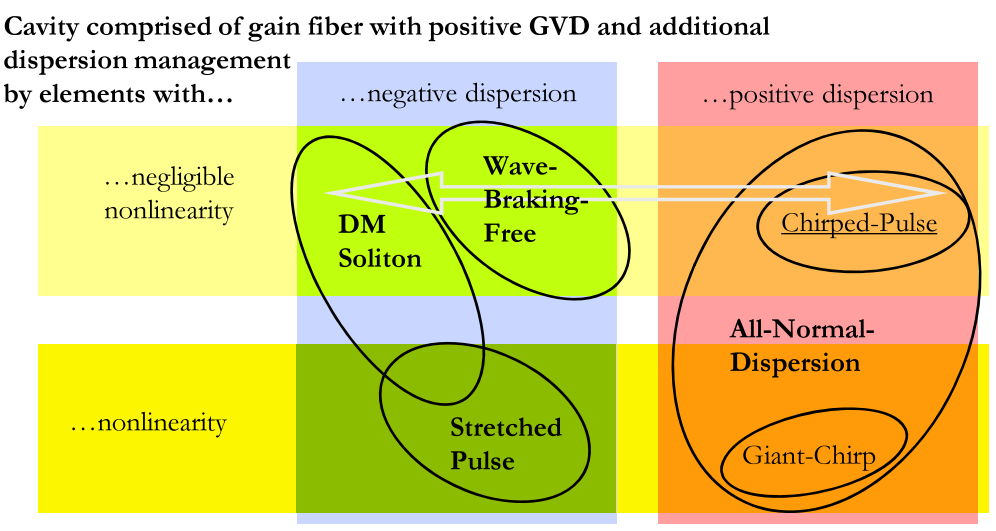

come accessible in the stretched-pulse laser [5, 6]. By further increasing the positive dispersion optical wave breaking can be avoided and the chirp remains positive throughout the whole cavity in the wave-breaking-free regime. High pulse energies are supported as the pulse is only partially compressed inside the laser using a dispersion compensating element with negligible nonlinearity like a chirped fiber grating or a bulk grating pair [7]. Most recent research focuses on all-normal-dispersion setups [8-18]. Without dispersion compensation, temporal shortening and thus a selfconsistent pulse evolution in these systems can be obtained just by strong self-amplitude modulation of the saturable absorber mechanism [8]. Furthermore, the temporal action of passive spectral filtering and gain filtering can significantly support pulse generation and stabilization in purely positive dispersion fiber lasers $[9,10]$. Due to the strong chirp and thus increased pulse length, highest pulse energies of several tens of nanojoules are supported [11]. Moreover, improved performance levels have been enabled by the use of low nonlinearity large-mode-area fibers [12-15]. Finally extreme stretching ratios in cavities containing long segments of passive fiber have been demonstrated in giantchirp oscillators $[16,17]$. These obtain high pulse energies, but pulse recompression becomes more and more challenging.

As the above overview reveals, the dispersion is one of the most detrimental parameters for pulse shaping in fiber lasers, besides drastically affecting the pulse dynamics, it also makes a direct impact on the laser performance. By adding a segment possessing large positive group delay dispersion (GDD) and no or negligible nonlinearity to an allnormal-dispersion fiber laser, a new mode-locked cavity design was proposed recently. This chirped-pulse oscillator was demonstrated in a fiber-integrated manner, using a chirped fiber Bragg grating (CFBG), which dominated the total cavity dispersion [18]. It was demonstrated that the local dispersion segment inside the cavity features a new aspect; however, up to now no systematic research of the pulse dynamics in the chirped-pulse regime has been reported.
In this letter we present a systematic experimental and numerical study on the influence of dispersion in a passively mode-locked fiber laser. While our emphasis is on the chirped-pulse regime at positive dispersion, a wider dispersion range is covered in this study. Stable mode-locked operation over a large net-cavity dispersion span, ranging from $-1.6 \mathrm{ps}^{2}$ to $+2.5 \mathrm{ps}^{2}$ is demonstrated at one laser setup. Strong pulse stretching is observed, showing that the application of large positive or negative GDD values could be one approach for further energy scaling. On the basis of the experimental results together with numerical simulations pulse shapes and varying intra-cavity dynamics are discussed.

\section{Experimental setup}

The passively mode-locked fiber laser is implemented in a linear cavity configuration (Fig. 2). A $1 \mathrm{~m}$ long piece of highly ytterbium doped (250 dB/m absorption@976 nm) polarization maintaining (PM) single-clad fiber with a core diameter of $6 \mu \mathrm{m}$ serves as gain medium. The fiber ends are angle-polished to eliminate parasitic reflection into the fiber or sub-cavity effects. A single-mode diode is used to core pump the fiber from one side through a short pass dichroic mirror. A half wave plate in combination with a polarization beam splitter (PBS1) allows to adjust the output coupling ratio, while a second polarizer (PBS2) ensures excitation of the slow axis of the PM fiber. Passive mode-locking is achieved employing a commercial highmodulation-depth semi-conductor saturable absorber mirror (SAM) with a fast relaxation time of $\sim 600 \mathrm{fs}$ as one of the cavity mirrors. A transmission grating-based stretcher or compressor is implemented on the other cavity end. Its lens and folding mirror are mounted on a translation stage, hence additional positive or negative dispersion can be continuously added by moving the stage by a distance $\pm g$ out of the zero-dispersion position. Thus, our cavity design allows tunability of the total cavity dispersion (TCD) over a large range between $+2.5 \mathrm{ps}^{2}$ and $-1.6 \mathrm{ps}^{2}$ without adding 
Fig. 2 Schematic representation of the passively mode-locked Yb-doped chirped-pulse fiber laser. SAM: saturable absorber mirror, $L$ : lens, $P B S$ : polarizing beam splitter, $H W P$ : half wave plate, $D C M$ : dichroic mirror, $H R$ : high reflector
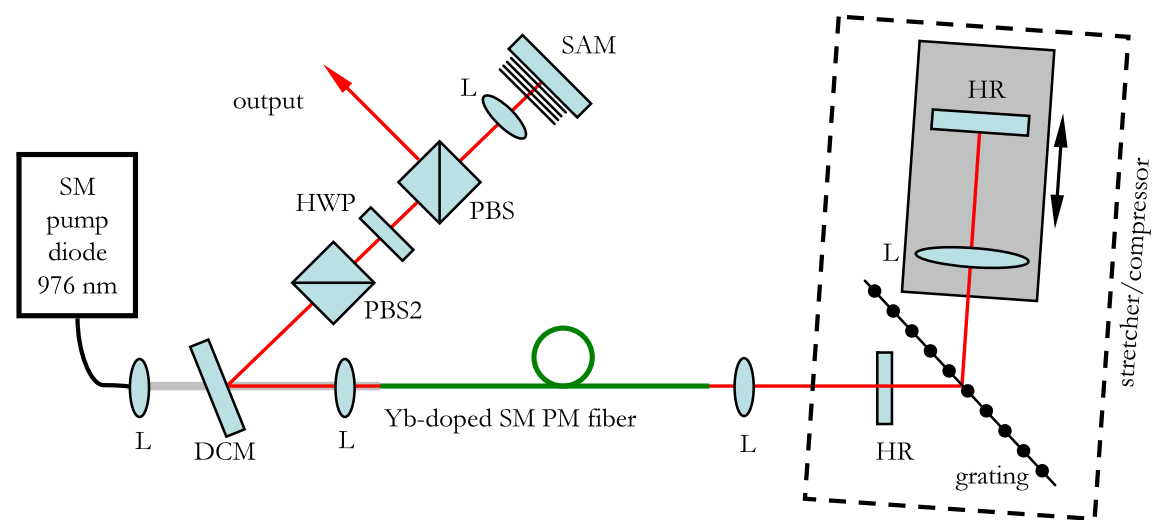

Fig. 3 Spectra and autocorrelation traces taken at the laser output for positive TCD values. (For quantitative evaluation of spectral and temporal widths see Fig. 10)
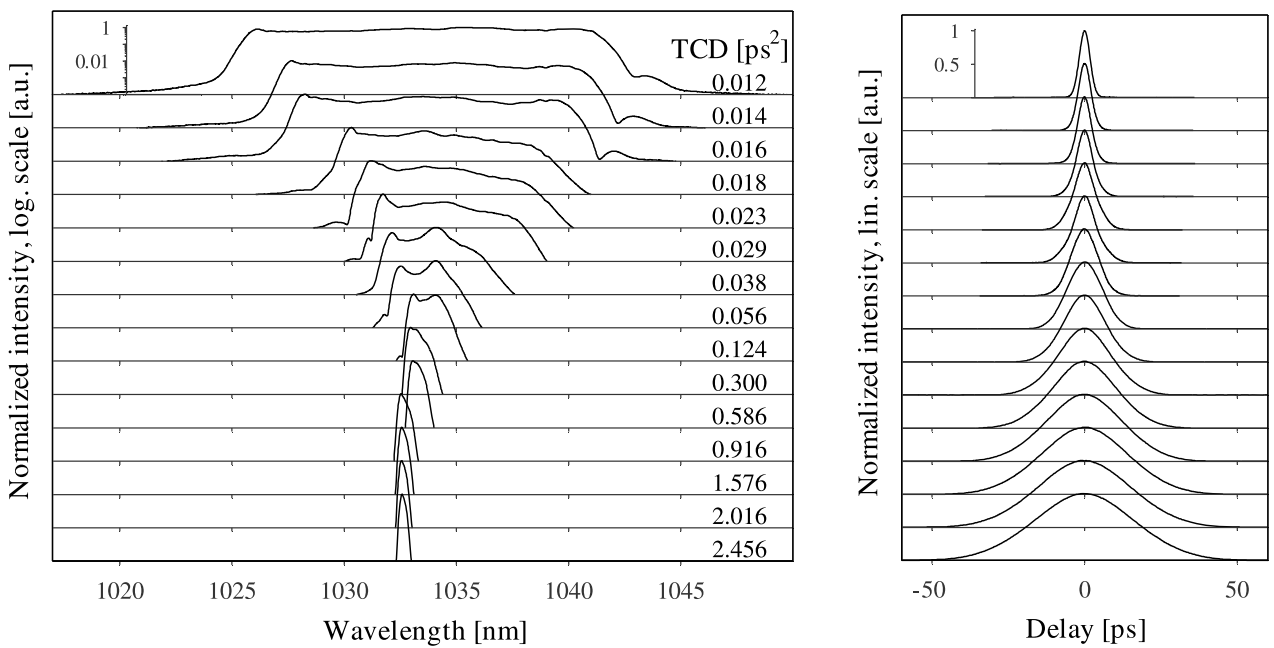

further material nonlinearity. When the stretcher is set to its zero-dispersion point the TCD resulting from the contributions of all other cavity components amounts $0.05 \mathrm{ps}^{2}$. For both intra-cavity dispersion control as for external pulse compression transmission gratings with a period of $800 \mathrm{~nm}$ are used. The polarization maintaining setup offers the advantage of a simplified theoretical description as it can be represented by a scalar model. Together with the continuously tunable dispersion management the system is an ideal platform to study the influence of dispersion on the pulse dynamics.

\section{Experimental results}

To adjust the saturation energy on the SAM the lens telescope was adapted until robust single-pulse mode-locked operation was obtained. At a pump power of about $250 \mathrm{~mW}$ and an output coupling ratio around $35 \%$ an amplitudestable pulse train with a repetition rate of $\sim 50 \mathrm{MHz}$ is observed using a $200 \mathrm{ps}$ rise time photodiode and a $200 \mathrm{MHz}$ analog oscilloscope. Single-pulse operation is additionally verified with a $150 \mathrm{ps}$ scan range autocorrelator.
3.1 Mode-locked operation at positive total cavity dispersion

Starting at one stable operation point slightly above zero netcavity dispersion, the total cavity dispersion (TCD) is varied (up to $2.5 \mathrm{ps}^{2}$ ) while only the intra-cavity power is slightly adjusted to secure saturation of the SAM at longer pulse durations. Figure 3 shows the optical spectra and autocorrelation traces of the emitted pulse train for different positive TCD values.

Spectrally broad operation $(15.7 \mathrm{~nm})$ is observed for small dispersion values $\left(0.012 \mathrm{ps}^{2}\right)$. The spectral width decreases continuously when the TCD is increased, resulting in a narrow bandwidth operation mode with bandwidths $(5 \mathrm{~dB})$ as small as $0.22 \mathrm{~nm}$ at highest accessed dispersion of $+2.5 \mathrm{ps}^{2}$. As can be seen from the autocorrelation traces, the pulse duration increases with dispersion. The intensity autocorrelations exhibit widths starting from $4.3 \mathrm{ps}$ at $0.012 \mathrm{ps}^{2}$ and resulting in up to $38.6 \mathrm{ps}$ at highest TCD.

Using a dispersive delay line consisting of two transmission gratings outside the cavity, the chirped output pulses were compressed close to their transform limited duration. The measured intensity autocorrelation traces produced by 
the compressed pulses are shown in Fig. 4 together with their corresponding transform limits, which were calculated from the intensity spectra, setting a zero spectral phase. The comparison shows that the pulses are well compressible both for low and high intra-cavity dispersion, demonstrating that the output pulses possess a near linear chirp. Small deviations from the transform limit are noticeable mainly in the pulse wings, but no dependence on TCD is visible. The large pulse stretching and the long pulse durations at positive stretcher dispersion prevent pulse breakup, hence up to maximum available pump power of $270 \mathrm{~mW}$ no double pulsing is observed.

\subsection{Mode-locked operation at negative total cavity dispersion}

Analog to the scenario at positive TCD, the oscillator is scanned through the negative TCD regime. Qualitatively similar behavior is observed in the negative dispersion region, where the spectral width $(5 \mathrm{~dB})$ decreases from $5.29 \mathrm{~nm} @-0.07 \mathrm{ps}^{2}$ to $0.64 \mathrm{~nm} @-1.6 \mathrm{ps}^{2}$. Whereas the autocorrelation durations increase from 0.8 ps to $3.9 \mathrm{ps}$. This

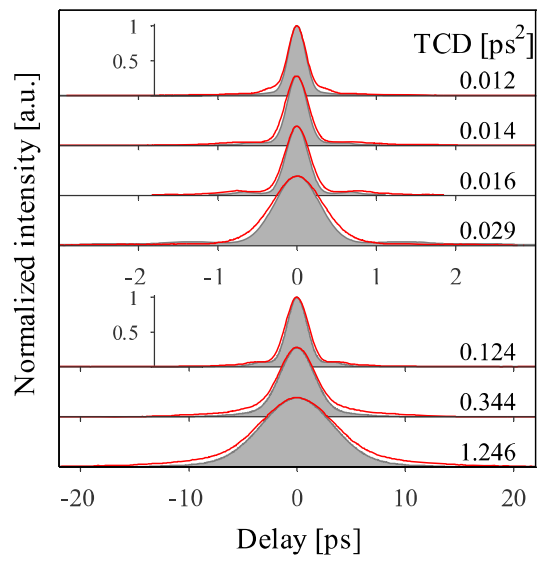

Fig. 4 Measured autocorrelation traces of the compressed pulses (red) together with the corresponding traces of the transform limit (gray) calculated from the spectra evolution is highlighted in Fig. 5. Kelly sidebands (KSB) [19] are visible in the spectrum. Although the gain fiber exhibits normal dispersion and negative dispersion is only included by the bulk grating setup, the laser clearly shows average soliton features.

Due to the non-uniform dispersion map the output pulses are not transform limited, but possess a negative chirp. External pulse compression is done with a second grating stretcher setup as used inside the cavity. The corresponding autocorrelation traces are shown in Fig. 6 together with their transform limits. The compressed durations vary from $0.5 \mathrm{ps}$ to $3.8 \mathrm{ps}$, respectively. It can be seen that the shorter pulses deviate slightly from the transform limit, especially in the wings. This is attributed to higher nonlinear effects due to the larger peak power, furthermore third-order dispersion plays a larger role close to zero net-cavity dispersion.

The laser is pumped with about $120 \mathrm{~mW}$ at negative TCD. Near zero-dispersion the oscillator operates in double pulse regime at high pump power. With longer pulse durations at large negative dispersion the peak power decreases and therefore nonlinear effects play a minor role where we obtained maximum output pulse energies (close to nanojoule) at maximum available pump power. This trend is additionally illustrated in Fig. 7 where the pump power thresholds for the transitions between single and double pulsing

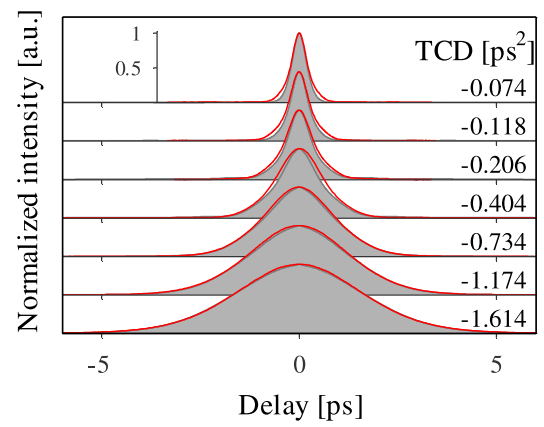

Fig. 6 Measured autocorrelation traces of the compressed pulses (red) together with the corresponding traces of the transform limit (gray) calculated from the spectra
Fig. 5 Spectra and autocorrelation traces taken at the laser output for negative TCD values. (For quantitative evaluation of spectral and temporal widths see Fig. 9)
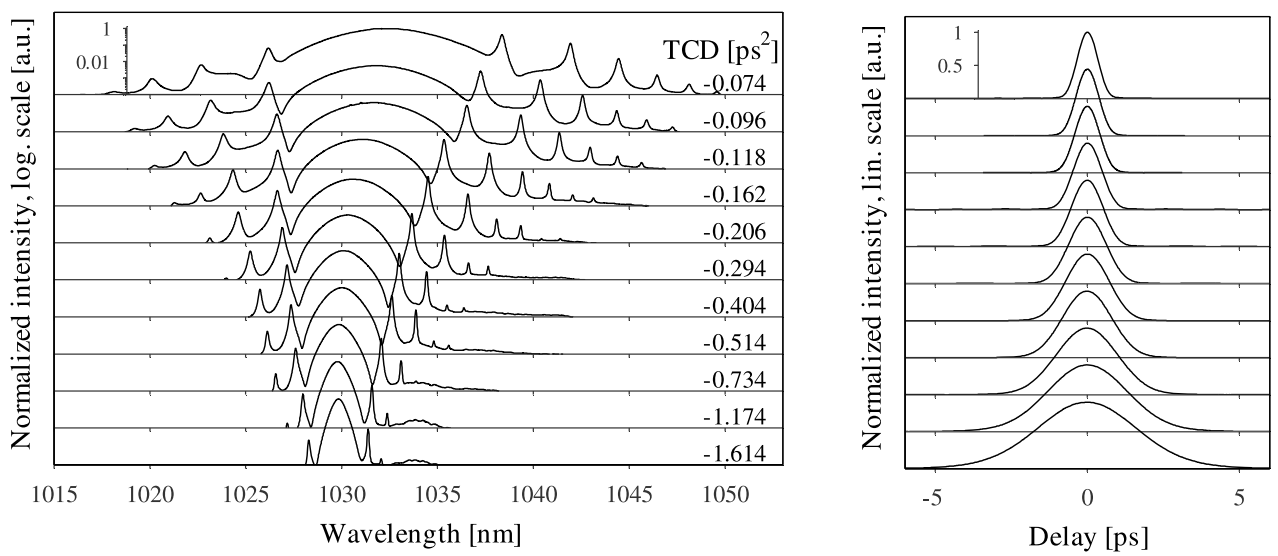
states are plotted (red and blue). The stability area for the single-pulse regime shifts significantly towards higher pump power with stronger dispersion.

\subsubsection{Evaluation of Kelly sidebands}

In reference [20] only fiber with negative dispersion is used inside the cavity and pulse propagation is described as average soliton, linking the KSB positions in the spectrum to the total cavity dispersion. From the soliton phase, taking only second-order dispersion into account the following dependency from the KSB frequency offset $\Delta \omega$ from the central frequency is derived:

$|N|=\frac{\mathrm{TCD}}{4 \pi}\left(\Delta \omega_{N}^{2}+\tau^{-2}\right)$

with $\Delta t=1.76 \tau$ being the FWHM pulse duration of the soliton and $N$ the KSB order. We apply a parabolic fit according to Eq. (1) with the parameters A and B as can be seen in Fig. 8(a) to our spectral data. The KSB positions and the fit curves are shown in Fig. 8(a) exemplary for two different dispersion settings of the intra-cavity grating compressor. As can be seen the data points are well modeled by the parabolas. From parameter A the TCD is calculated for each compressor setting. The results are shown

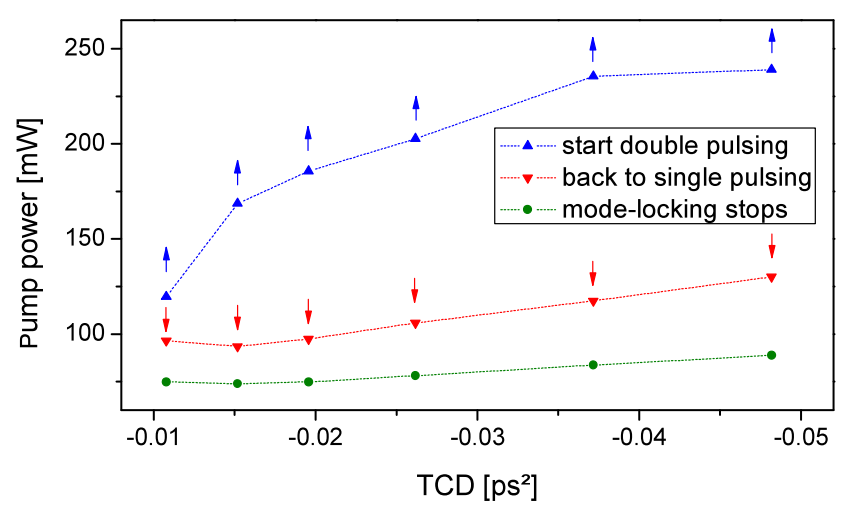

Fig. 7 Critical pump power for the change from single to double pulse operation (blue), the change from double to single-pulse operation (red) and loss of mode-locking (green) over TCD in Fig. 8(b) and good agreement with the values calculated from the compressor geometry is observed. Interestingly the average soliton model yields sensible agreement despite the fact that only normal-dispersive fiber is used in our setup. To stress this further we additionally calculated the pulse duration from fit parameter B together with Eq. (1) and compare it in Fig. 8(c) with the pulse duration obtained from the autocorrelation traces measured in the experiment. One can see that the pulse duration is in average slightly underestimated, but still good agreement is obtained.

\subsection{Dependencies of pulse parameters on dispersion}

The dependencies of the pulse parameters (spectral and temporal widths) of the chirped and compressed pulses on TCD are summarized in Fig. 9(a) for the case of negative dispersion. As can be seen, the pulse duration increases approximately linearly with dispersion whereas the decrease in spectral width seems to saturate quickly. The duration of the compressed pulses (green, $x$ ) approaches the duration of the chirped pulses (green, $\bullet$ ) for large dispersion values and hence the compression factor (Fig. 9(b)) approaches one asymptotically. This is a result of the narrow spectral bandwidth at high dispersion, which, despite nearly linearly increasing chirp (red, $\bullet$ ), prevents effective pulse stretching.

Figure 10 displays the pulse parameter evolution with dispersion for positive TCD. The pulse duration (Fig. 10(a)) increases rapidly with increasing dispersion especially for smaller dispersion values. The slope is much steeper over the whole range compared to the anomalous dispersion regime, as with decreasing influence of the dispersion compensation and finally its omission there is no pulse shortening mechanism besides the saturable absorber in the cavity. Nevertheless, the effective pulse stretching is attenuated also here by the decreasing spectral width. The compression factor (Fig. 10(b)) therefore shows a qualitatively similar behavior as in the case of negative dispersion, but reaches much higher values. Whereas pulses at $0.01 \mathrm{ps}^{2}$ can be compressed down to a fifteenth of their initial duration, the chirped pulses at $2.5 \mathrm{ps}^{2}$ possess only three times the duration of their transform limit. Longer pulse durations lower

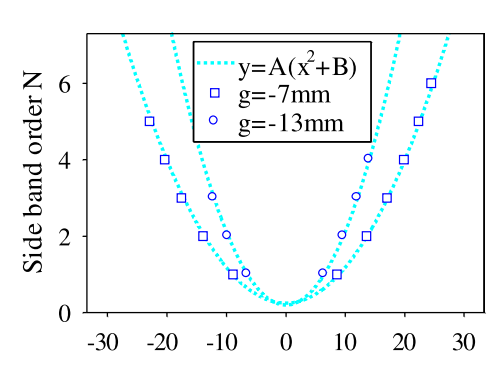

a Angular frequency offset $\left[\mathrm{Thz}^{2}\right]$

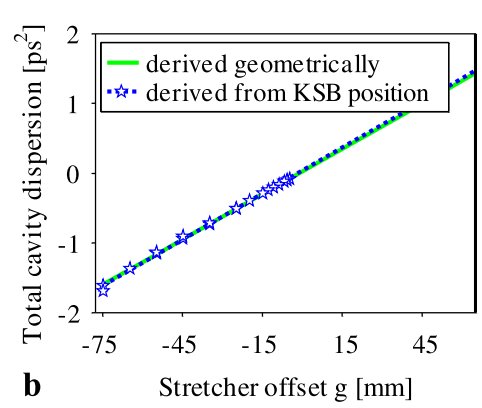

Fig. 8 (a) KSB order over frequency offset with parabolic fit; (b) computed TCD from fit parameter A over stretcher position g; (c) computed pulse duration from fit parameter B over pulse duration measured with AC 


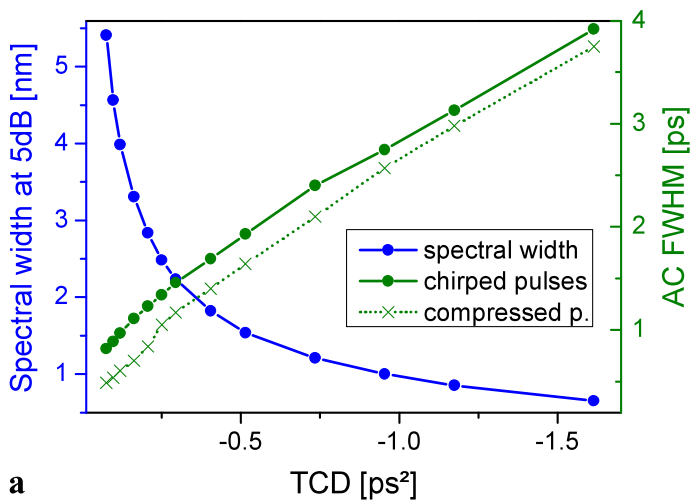

Fig. 9 Evolution of pulse parameters with TCD. (a) spectral (blue) and temporal pulse width (green, •), pulse duration of externally compressed pulses (green, $\times$ ); (b) compression factor (green, $\bullet$ ) and
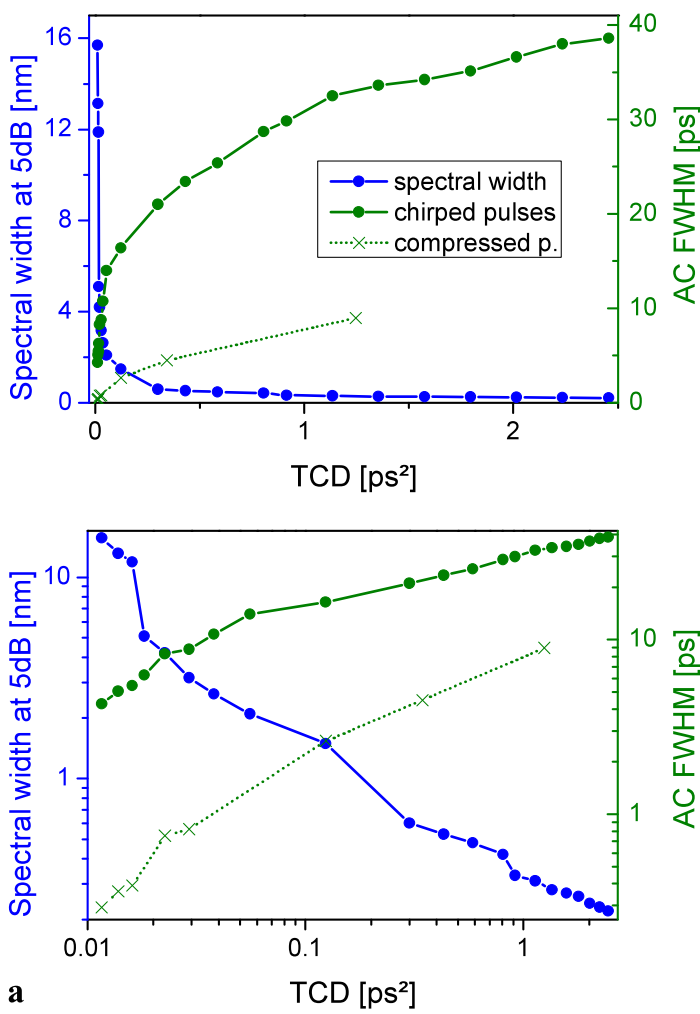

Fig. 10 (a) Spectral (blue) and temporal (green, •) pulse width over dispersion. Pulse duration of the externally compressed pulses. (green, $\times$ ). Top: linear, bottom: logarithmic scale. (b) Compression factor

the peak power and consequently weaken self-phase modulation. Furthermore the spectral broadening effect of SPM is reduced for highly chirped pulses, resulting in decreased spectral widths. Therefore the increase in pulse duration saturates for large GDD values. The external dispersion needed for pulse compression (Fig. 10(b)) increases, also qualitatively similar to the negative case, near linearly with internal dispersion. The slope, however, is more than 50 times

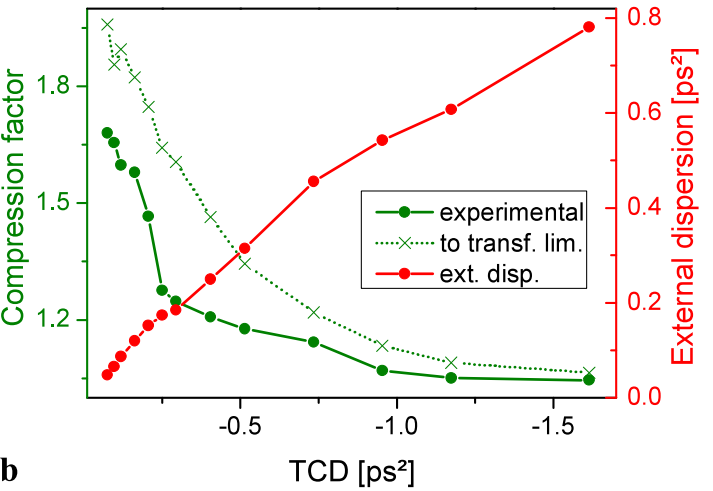

external dispersion needed for pulse compression ( $r e d$ ), for comparison theoretical compression factor to transform limit $($ green,$\times)$
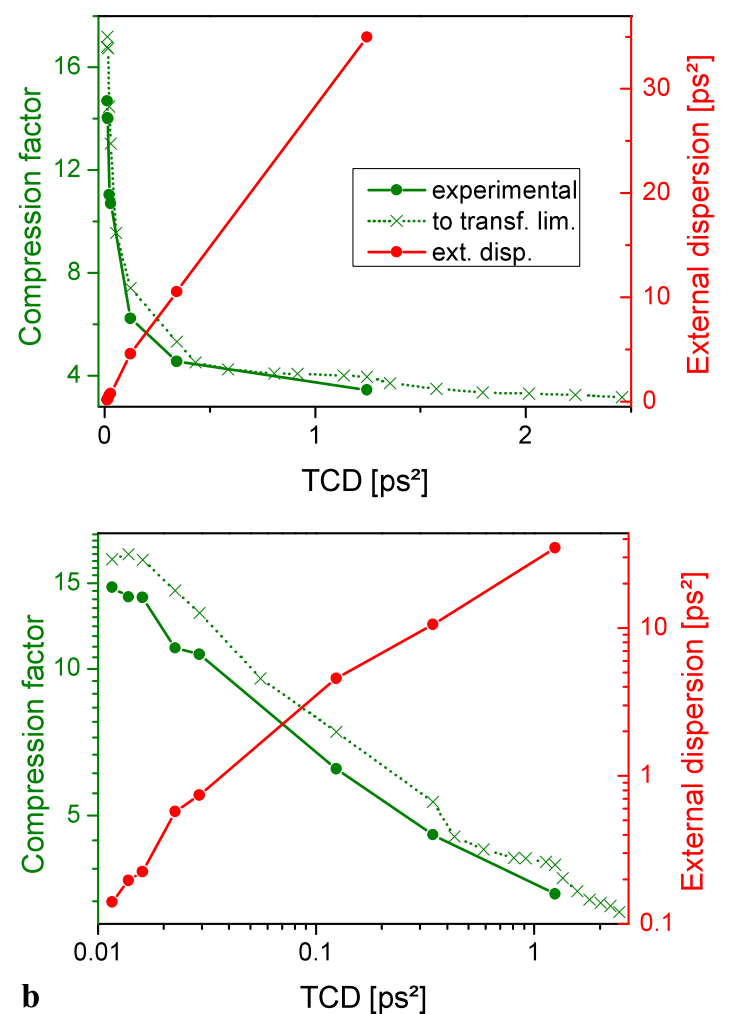

$($ green,$\bullet)$ and required amount of external dispersion $($ red $)$ over TCD. For comparison theoretical compression factor towards transform limit (green, $\times$ ). Top: linear, bottom: $\log$. scale

steeper, thus grating distances of up to several meters were necessary for pulse compression.

Comparing the decrease in spectral bandwidth we can see that for similar absolute values of dispersion the spectral width decreases three times slower for positive total cavity dispersion than in the case of negative dispersion. This slow decrease despite at the same time faster increasing pulse durations is caused by the fact that SPM has a spectral broad- 

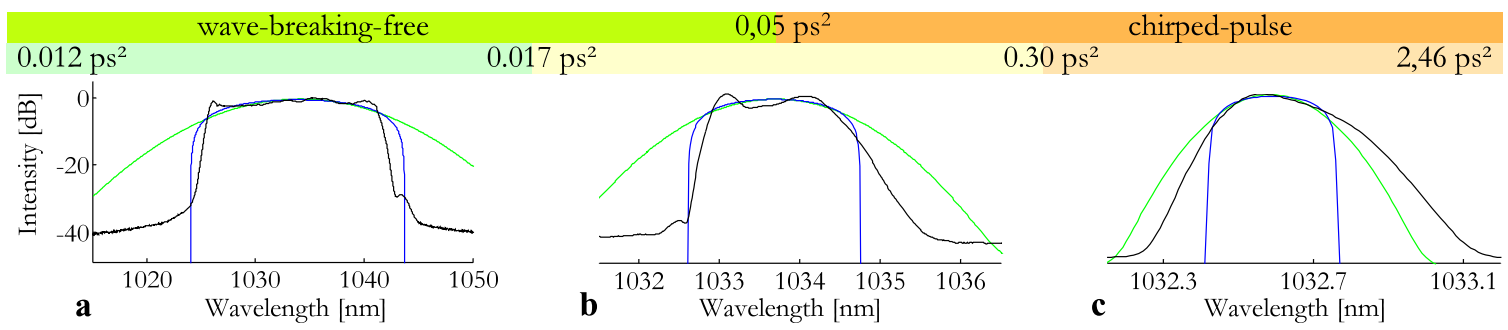

Fig. 11 Spectra (measured-black) for $(\mathbf{a}) \mathrm{TCD}=0.012 \mathrm{ps}^{2}$, (b) $\mathrm{TCD}=0.124 \mathrm{ps}^{2}$, (c) $\mathrm{TCD}=1.80 \mathrm{ps}^{2}$. Fit curves for Parabola $($ blue $)$ and Gaussian (green)
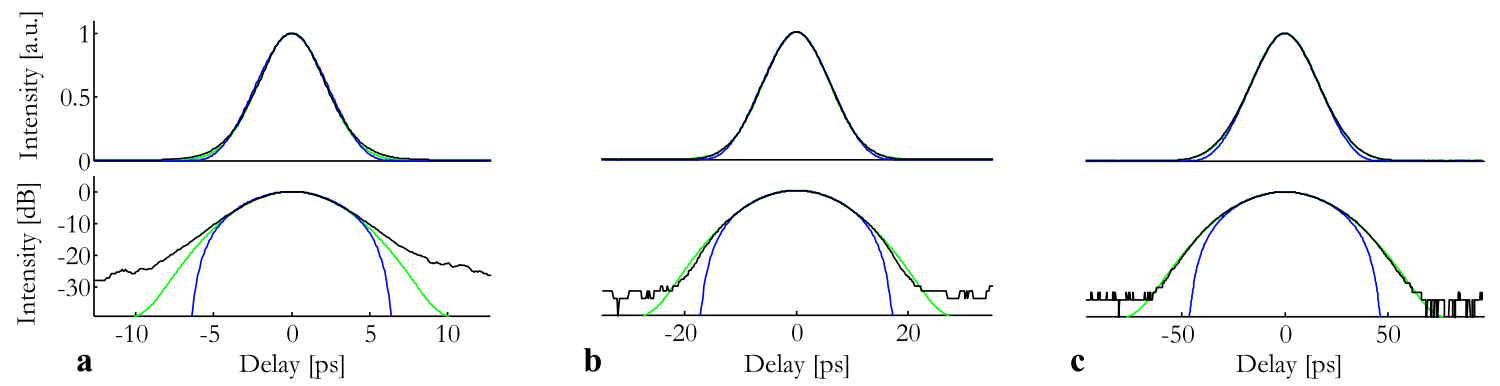

Fig. 12 AC traces (measured-black) for the representative cases (a) TCD $=0.014 \mathrm{ps}^{2},(\mathbf{b}) \mathrm{TCD}=0.056 \mathrm{ps}^{2},(\mathbf{c}) \mathrm{TCD}=2.46 \mathrm{ps}^{2}$, with the corresponding fit curves for Gaussian (green) and parabolic pulses (blue), top: lin. scale, bottom: log. scale

ening effect at all cavity positions as the pulse is always positively chirped throughout the round trip, in contrast to the case at negative dispersion, leading to completely different dynamics inside the resonator.

\subsection{Pulse shape}

The pulse shape changes significantly with varying dispersion, as is already visible in Figs. 3 and 5. In the range of 0.012 to $0.016 \mathrm{ps}^{2}$ the spectra exhibit steep flanks, caused by a combination of strong SPM and spectral shaping by the gain profile. With temporal broadening of the pulses and decrease in spectral width these effects lose force with increasing dispersion. Consequently the spectral flanks get flatter. To illustrate this, a representative spectrum for each of the dispersion ranges (a) below $0.017 \mathrm{ps}^{2}$, (c) above $0.3 \mathrm{ps}^{2}$ and (b) the transition between the two is plotted in Fig. 11. For comparison a Gaussian and a parabolic least-squares fit is shown with each spectrum. The spectra exhibit an asymmetry which is attributed to the asymmetric temporal response of the saturable absorber acting on the chirped pulses. As the longer spectral tails at higher dispersion are much closer to a Gaussian shape it is not surprising that the temporal shape (Fig. 12) in case (c) is well modeled by a Gaussian fit curve. For small dispersion values the pulse wings get more pronounced (a), but the central part still has a good overlap with a Gaussian shape.

Over the whole negative dispersion range the temporal pulse shape fits well with the corresponding Gaussian fit curves. Especially the pulse wings show Gaussian decline whereas the slowly falling $\operatorname{sech}^{2}$ function overestimates the experimental curves in this region (exemplary Fig. 13). Shapes of spectral intensity are also very close to a Gaussian, only for very high negative dispersion the spectral shape approaches the $\operatorname{sech}^{2}$ characteristic. Thus at negative TCD the laser exhibits both soliton features (KSB) as well as stretched-pulse attributes (Gaussian shape) similar as reported in [4] with an erbium fiber laser.

\section{Numerical simulation}

To gain further insight especially into intra-cavity dynamics, we performed numerical simulations, using the same nondistributed model based on the nonlinear Schrödinger equation as used in [12], where all cavity elements are treated separately. The simulation scheme of the linear cavity is depicted in Fig. 14, the parameters were chosen to match the experimental parameters. Starting from noise, the simulations were continued until the field settled to a stable solution.

Leaving all parameters fixed, only the amount of dispersion applied by the stretcher element is varied for the both cases of positive and negative TCD. The stable solutions for the representative cases (spectrum and autocorrelation traces) are shown in Figs. 15 and 16 together with the corresponding experimental results. Naturally the simplified model does not reproduce every spectral modulation 
Fig. 13 AC (left) and spectrum (right) of the pulses (measured-black) at $-0.21 \mathrm{ps}^{2}$. For comparison the corresponding curves for a sech $^{2}$ pulse (red) and a Gaussian pulse (green)

Fig. 14 Cavity scheme used for the simulations

Fig. 15 Comparison of spectra and autocorrelation traces obtained from simulations (red) and experiment (gray) for positive dispersion
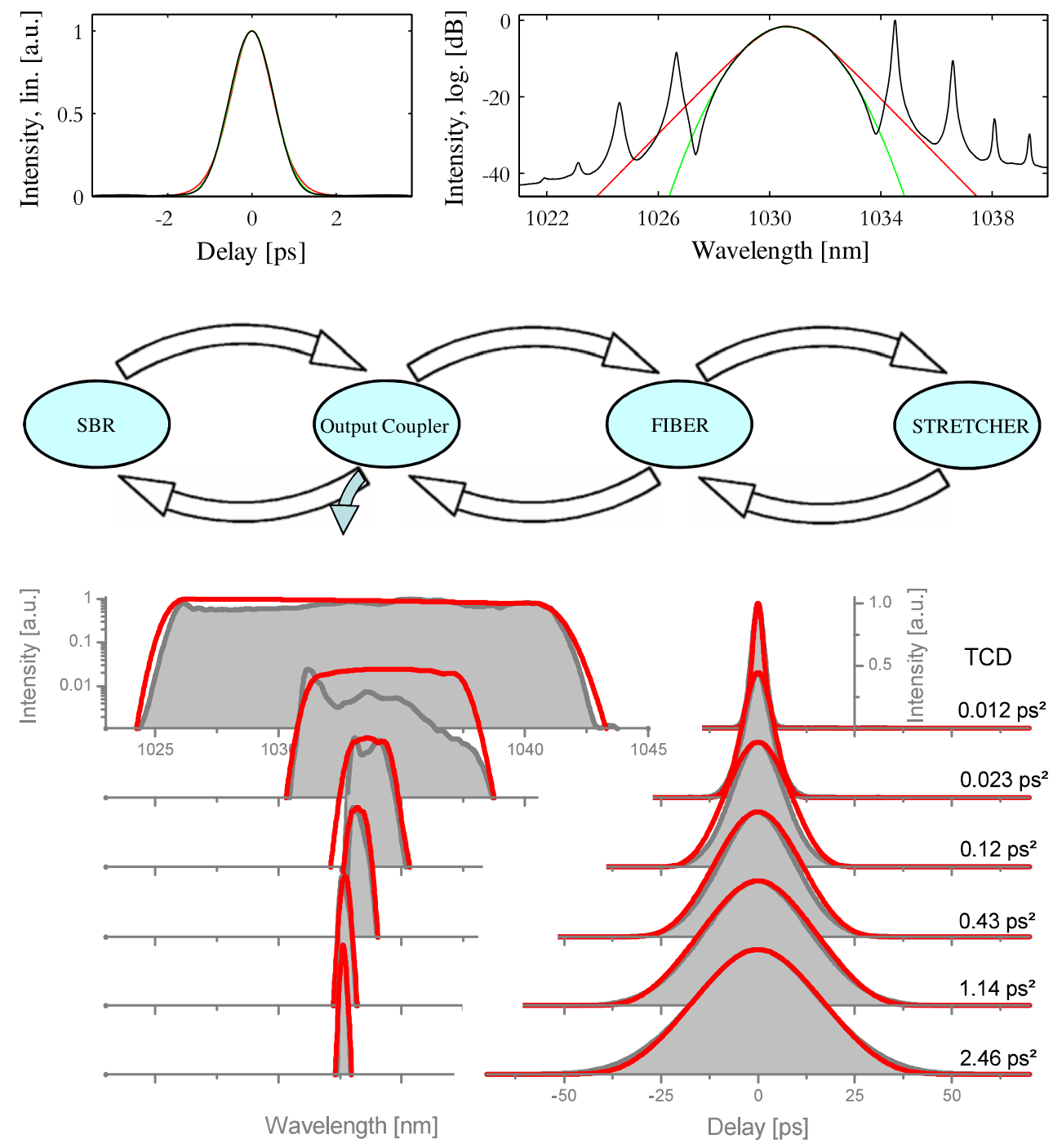

Fig. 16 Comparison of spectra and autocorrelation traces obtained from simulations (red) and experiment (gray) for negative dispersion

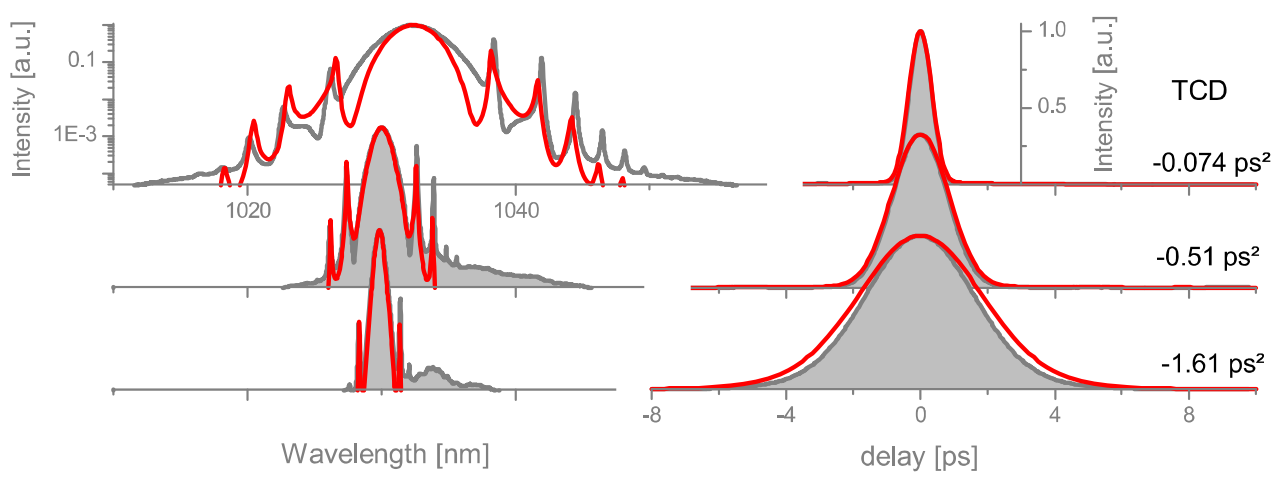

or asymmetry, but in general a good agreement of the pulse width and the principal shape is obtained over the whole dispersion range, proving the suitability of the applied model for further investigations.

The simulation easily allows one to extend the experimentally accessed dispersion range. This was done straight forward by increasing and decreasing the dispersion, start- ing always from a random noise field distribution. Only very close to zero net-cavity dispersion the simulation is started from an initial pulse to exclude double pulse solutions. Having a look at the evolution of the pulse width parameters with dispersion in Fig. 17, reveals a change in the slope of the pulse duration around the zero-dispersion-position of the stretcher $\left(\mathrm{TCD} \approx 0.05 \mathrm{ps}^{2}\right.$ ), which marks the transi- 
Fig. 17 Evolution of pulse parameters over TCD obtained from numerical simulations, spectral width (blue) and chirped ac pulse duration (green) in log. scale, the area accessed experimentally is marked gray

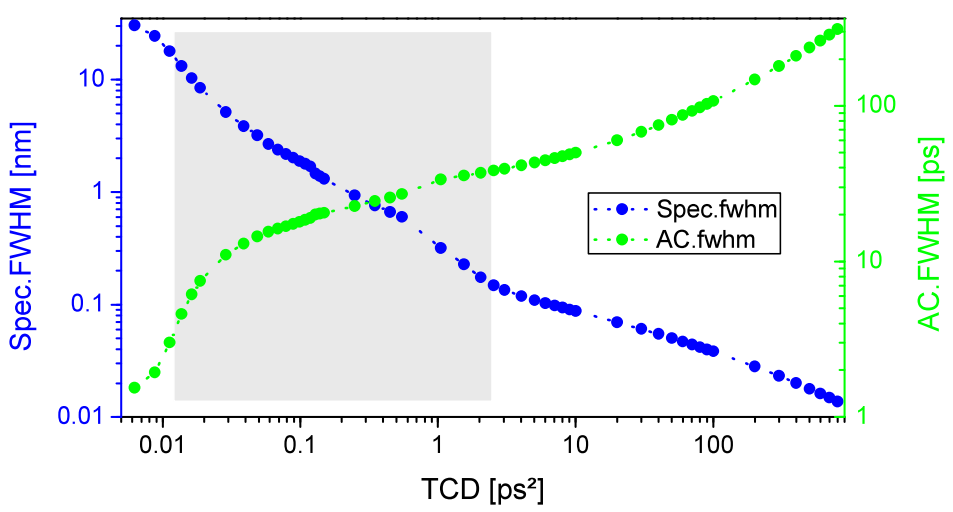

loss inside the cavity. This could be used to control the chirp of the output pulses without changing the position of the output coupler.

The evolution of the pulse duration changes significantly with TCD. For highly negative GDD settings (Fig. 18(c)), the pulses are no longer shortened (Fig. 18(a, b)), but stretched by the "compressor". The dispersive stretching during the first fiber passage and the temporal compression as a consequence of the spectral narrowing during the second passage lose influence with increasing negative dispersion. Finally at large negative TCD (c) the temporal dynamic is dominated by the compressor and the saturable absorber action.

The scenario at positive TCD is illustrated in Fig. 18(d-f). At a total cavity dispersion below $0.05 \mathrm{ps}^{2}$ (Fig. 18(d)) the material dispersion is partially compensated and the pulse duration features the for the wave-breaking-free regime characteristic minimum directly after the compressor. The chirp is positive along the whole resonator and hence the pulse experiences monotonic dispersive broadening in the fiber sections. In the spectral domain the pulse is narrowed by the gain profile in the beginning of the fiber sections, but later nonlinear broadening dominates at higher pulse energy. Finally the chirped pulse is both shortened in temporal and spectral domains by the saturable absorber.

Increasing the dispersion (Fig. 18(e, f)), the dispersion control changes its sign and the temporal evolution becomes strictly monotonic reaching the chirped-pulse regime. Due to the longer pulses the spectrum experiences no significant broadening and hence the gain bandwidth does no longer shape the spectrum. In consequence also the spectral width evolves monotonically. Clearly visible is the chirp decreasing effect of SPM on the highly positively chirped pulses. At very large TCD (Fig. 18(f)) the stretcher and the SA dominate the temporal evolution as the fiber dispersion becomes negligible, accordingly the local temporal stretching feature characteristic for the chirped-pulse regime becomes prevailing. For maintaining a self-consistent evolution in the highly chirped pulse regime, the fiber nonlinearity strongly helps to compensate the dispersive chirp, while obviously the SA by tailoring the nonlinearity, e.g. by changing the gain and 


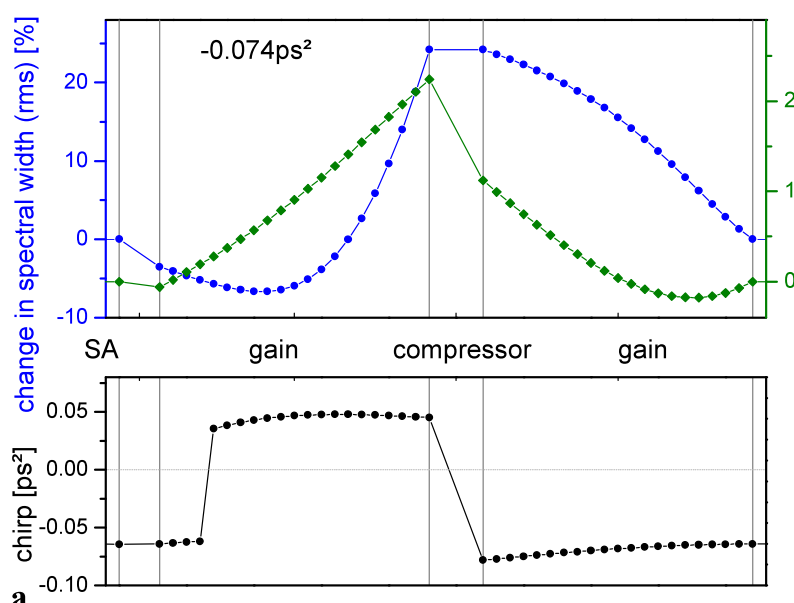

a
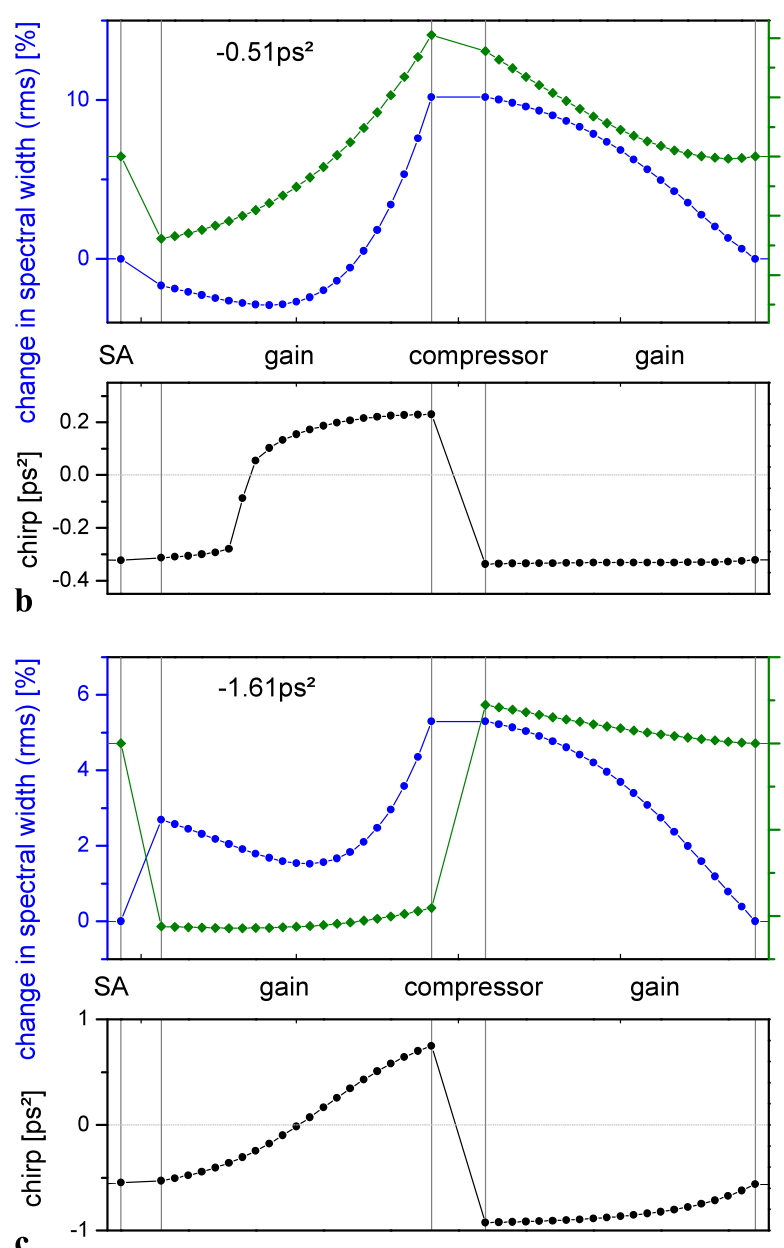
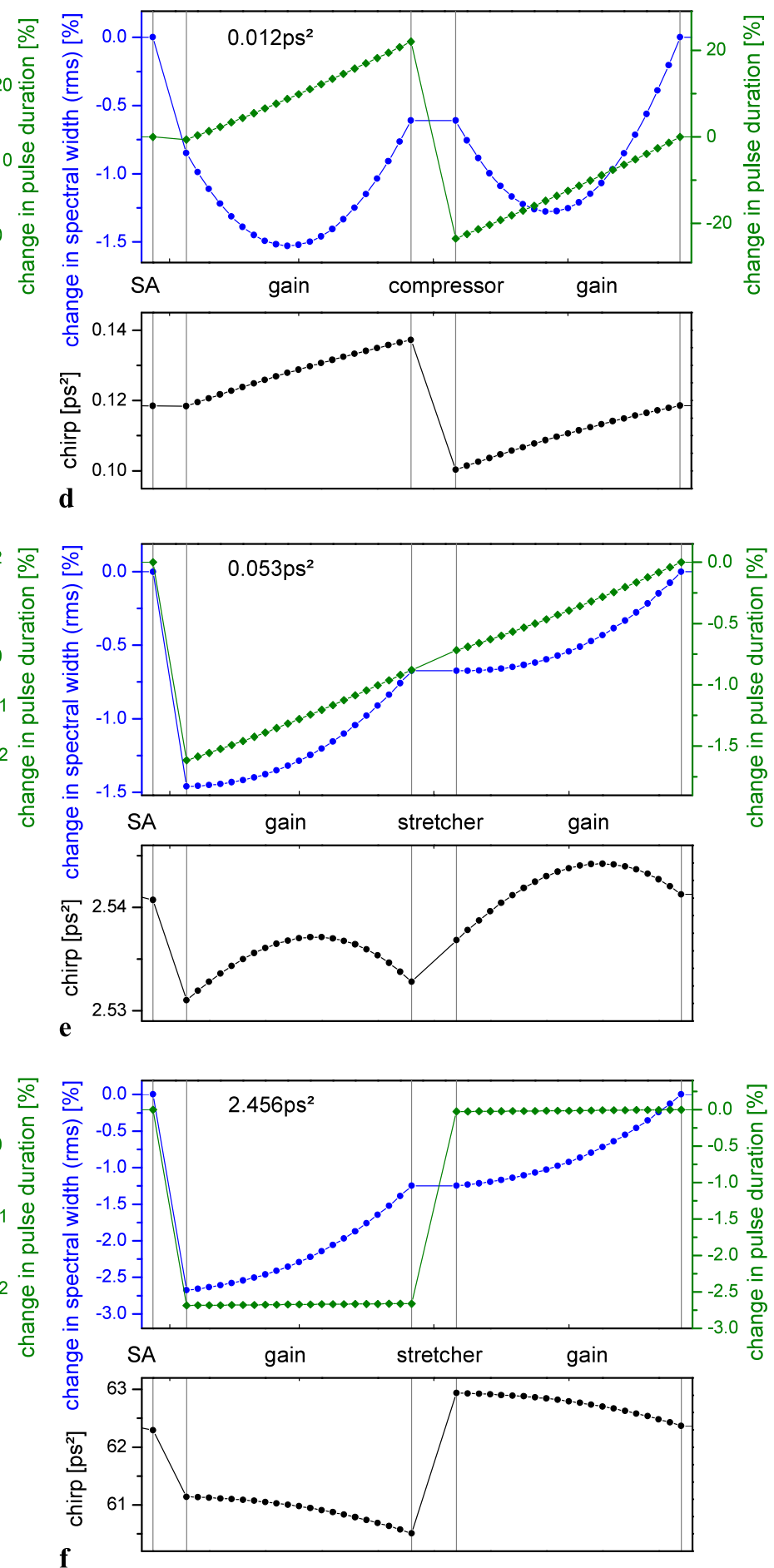

Fig. 18 Change in spectral width (blue) and pulse duration (green) along the cavity and evolution of chirp (black) for (a-c) negative and (d-f) positive TCD

plays the key role in the spectral and temporal domains as it represents the only pulse shortening mechanism in the cavity.

Note the different evolutions of the chirp for the cases in Fig. 18(d-f). In the WBF regime (Fig. 18(d)) the chirp is ruled by the alternating dispersion and evolves linearly in the positive fiber and negative compressor element, the SA has little influence. By contrast the behavior appears inversed in the highly chirped-pulse regime (Fig. 18(f)). Due to the inversed sign of the dispersion control the chirp is increas- 

over TCD, spectral (blue) and temporal (green) domain
Fig. 19 Pulse breathing ratio during one resonator round trip
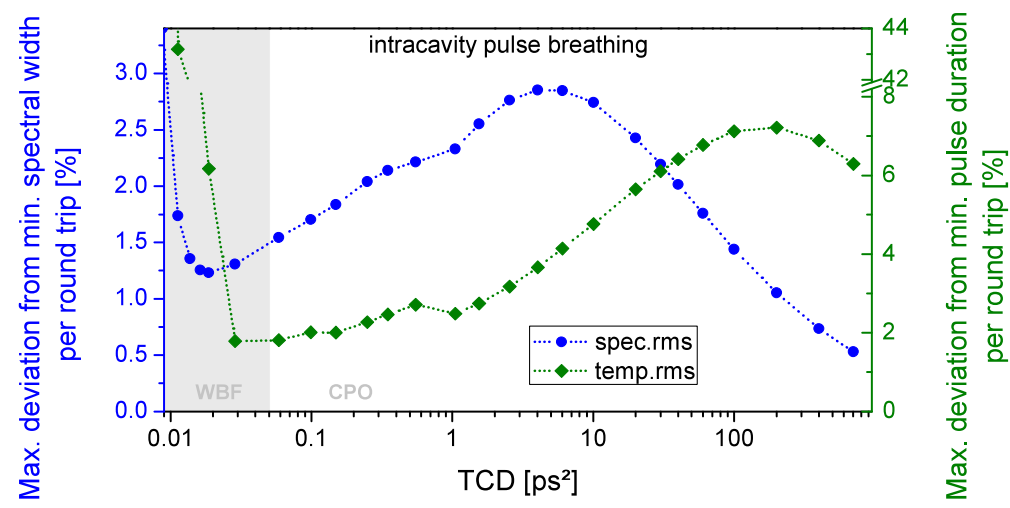

ing in the stretcher, whereas it is decreasing with increasing SPM in the fiber sections. The third case appears in the transition area between the regimes (Fig. 18(e)), where the chirp is initially increasing in the fiber segments and is then partially compensated by SPM within the same fiber passage.

The pulse breathing inside the cavity is clearly largest close to zero cavity dispersion as the pulses in this range are spectrally broad and thus susceptible to dispersion variations and at the same time their short durations imply strong spectral breathing due to SPM. Indeed, as can be seen in Fig. 19, the breathing ratio decreases drastically with increasing TCD in the WBF regime. In the chirpedpulse regime, however, despite the in general relatively low breathing ratios, the intra-cavity pulse breathing increases first of all with dispersion before it drops at extremely high TCD values.

\subsection{Pulse energy scaling}

In the above simulations a reduction in the accumulated nonlinear phase per round trip of two orders of magnitude is observed with increasing TCD, which is what one would expect from the increase in pulse duration. To investigate the basic pulse energy scaling behavior within the chirped-pulse approach, the simulation was started again with the same parameters as before, but this time the saturation energy of the gain was varied. For each dispersion setting the simulation was started from noise and every 1500 round trips the saturation energy was increased by $10 \%$ until pulse breakup was observed. The saturation energies leading to pulse breakup are summarized in Fig. 20 for the different TCD values. The trend in Fig. 20 clearly visualizes the potential of the chirped-pulse approach for energy scaling. Stable pulse solutions beyond $100 \mathrm{~nJ}$ exist in our linear cavity design using standard fiber dimensions. However, a more complete picture of the pulse energy limitations in chirped-pulse fiber lasers would require a detailed study of the influence of all important parameters in a more extensive set of simulations. The effect of an increased modulation depth [21] is of particular interest for energy scaling in this regime. This issue

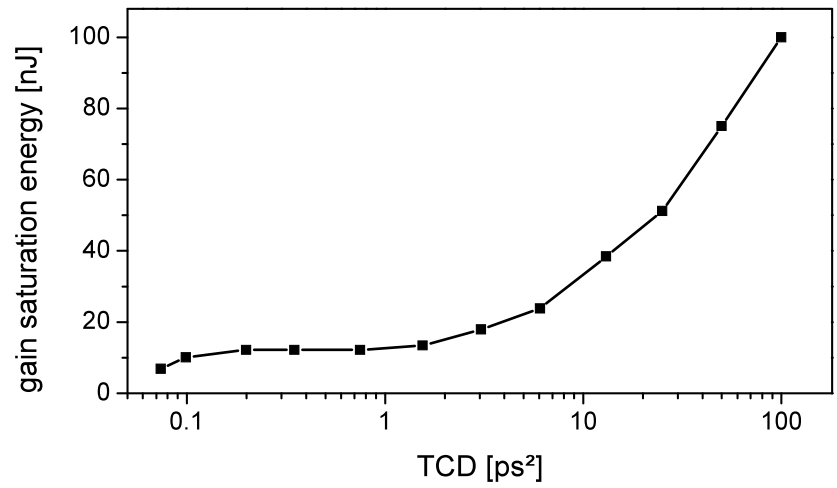

Fig. 20 Pulse energy scaling for the investigated cavity configuration: the pump power is increased stepwise, the graph shows the gain saturation energy for which pulse breakup is observed for each TCD

and also the influence of additional spectral filtering, different starting conditions and cavity configurations need further investigation.

\section{Conclusion}

A detailed study on the impact of different intra-cavity GDD values on the pulse dynamics in chirped-pulse fiber lasers is presented for the first time. Stable mode-locked operation over a large total cavity dispersion range from -1.6 to $+2.5 \mathrm{ps}^{2}$ is demonstrated in one oscillator setup, allowing for direct observation of the evolution of pulse parameters also across the different fiber laser regimes. Pulses exhibit average soliton features at negative TCD despite the solely positive fiber dispersion in the cavity. Longer pulses at larger dispersion values lower the peak power and thus allow to raise double pulsing threshold. Pulse durations increase quickly with higher dispersion especially at positive net-cavity dispersion. Thus strongly chirped pulses with long pulse durations (several tens of ps) and decreased bandwidths due to decreased nonlinearities are obtained at large TCD. Intra-cavity pulse evolution is discussed along with numerical simulations, highlighting the characteristic dy- 
namics in the different regimes with the strong local temporal stretching feature in the chirped-pulse regime. The simulation furthermore confirms that the application of large positive GDD values could be one approach for further energy scaling by restraining nonlinear effects due to the reduced peak power.

Acknowledgements This work was partly supported by the German Federal Ministry of Education and Research (BMBF) under contract 13 N10773 as well as the Inter Carnot \& Fraunhofer program under project APUS. M. Baumgartl acknowledges support from Carl-ZeissStiftung.

\section{References}

1. J.W. Nicholson, M. Andrejco, Opt. Express 14, 8160 (2006)

2. B. Ortaç, M. Plötner, T. Schreiber, J. Limpert, A. Tünnermann Opt. Express 15, 15595 (2007)

3. I.N. Duling III, Opt. Lett. 16, 539 (1991)

4. K. Tamura, L.E. Nelson, H.A. Haus, E.P. Ippen, Appl. Phys. Lett. 64, 149 (1994)

5. H.A. Haus, K. Tamura, L.E. Nelson, E.P. Ippen, IEEE J. Quantum Electron. 31, 591 (1995)

6. L.E. Nelson, S.B. Fleischer, G. Lenz, E.P. Ippen, Opt. Lett. 21, 1759 (1996)
7. F.Ö. Ilday, J.R. Buckley, H. Lim, F.W. Wise, W.G. Clark, Opt. Lett. 28, 1365 (2003)

8. R. Herda, O.G. Okhotnikov, IEEE J. Quantum Electron. 40, 893 (2004)

9. A. Chong, W.H. Renninger, F.W. Wise, J. Opt. Soc. Am. B 25, 140 (2008)

10. L.M. Zhao, D.Y. Tang, J. Wu, Opt. Lett. 31, 1788 (2006)

11. A. Chong, W. Renninger, F. Wise, Opt. Lett. 32, 2408 (2007)

12. M. Baumgartl, B. Ortaç, C. Lecaplain, A. Hideur, J. Limpert, A. Tünnermann, Opt. Lett. 35, 2311 (2010)

13. B. Ortaç, M. Baumgartl, J. Limpert, A. Tünnermann, Opt. Lett. 34, 1585 (2009)

14. C. Lecaplain, B. Ortaç, G. Machinet, J. Boullet, M. Baumgartl, T. Schreiber, E. Cormier, A. Hideur, Opt. Lett. 35, 3156 (2010)

15. M. Baumgartl, F. Jansen, F. Stutzki, C. Jauregui, B. Ortaç, J. Limpert, A. Tünnermann, Opt. Lett. 36, 244 (2011)

16. W.H. Renninger, A. Chong, F.W. Wise, Opt. Lett. 33, 3025 (2008)

17. D.-F. Liu, X.-J. Zhu, C.-H. Wang, J.-J. Yu, E.-X. Fang, J.-J. Wang, Laser Phys. 21, 414 (2011)

18. B. Ortaç, M. Plötner, J. Limpert, A. Tünnermann, Opt. Express 15, 16794 (2007)

19. S.M.J. Kelly, Electron. Lett. 28, 806 (1992)

20. M.L. Dennis, I.N. Duling III, IEEE J. Quantum Electron. 30, 1469 (1994)

21. C. Lecaplain, M. Baumgartl, T. Schreiber, A. Hideur, Opt. Express 19, 26742 (2011) 